\title{
Which dogs bite?
}

\author{
P. JARRETT
}

Accident and Emergency Department, St James's University Hospital, Leeds

\section{SUMMARY}

Young children (less than 11 years old) are a particular risk group for dog bites. Dog bites commonly occur from the family pet. Alsatian or alsatian mixes are the biggest group in the study causing dog bites. Alsations are a popular breed. By comparison Retrievers (Labrador and Golden), also a popular breed, caused few bites.

\section{INTRODUCTION}

There has recently been public concern about attacks caused by certain species of dogs. This is a study of patients presenting to an Accident and Emergency department who had been bitten by a dog. The purpose was to find out how and where patients came to be bitten and which species of dogs were involved.

\section{SUBJECTS METHODS AND RESULTS}

Patients who presented to the Accident and Emergency Department between April and July 1989, and who were seen by the author, were each asked a series of questions from a standard questionnaire. They were invited to pick out the dog that had bitten them from a selection of pictures of common dogs, to ensure accuracy in reporting.

A total of 75 patients with dog bites were seen, the ratio of male to female patients being 1.9:1. Twenty-four (32\%) were children less than 11 years old. Forty-nine people $(65 \%)$ had seen or met the dog before. Of these 49 people, 20 $(41 \%)$ were bitten by their family dog (18 in their own home) and $18(37 \%)$ were bitten by a neighbour's dog. 
There were a total of 81 wounds. The upper limb and hand received a total of 34 wounds $(42 \%)$. The lower limb had 30 wounds $(37 \%)$. The face received 7 wounds $(9 \%)$. Five wounds $(6 \%)$ required suturing.

The number of bites caused by different species were recorded (Table 1).

All patients who claimed to have been bitten by Alsatians, Rottweilers, Dobermanns and Retrievers accurately identified the species from the pictures.

Of those bitten, 36 people $(48 \%)$ claimed an unprovoked attack, including 9 of the children less than 11 years old (37\% of that age group).

There were 19 Alsatian bites, and 15 of these $(79 \%)$ were claimed to be unprovoked. Unprovoked bites from other breeds combined were 21 of 56 bites (37\%). Fifteen $(20 \%)$ of the bites occurred while playing with a dog and only $6(8 \%)$ occurred while stopping a dog fight.

The total number of pedigree registrations to the Kennel Club 1979 to 1988 and the number of new registrations in 1988 were used as a guide to the popularity of the different dog species (Table 1).

\section{COMMENT}

Many people were bitten by dogs that they knew. The bites commonly occur in the home from the family pet.

Children under 11 years old were at particular risk. This age group accounted for $32 \%$ of the bites, but form $14 \%$ of the total population of the U.K. (HMSC 1989). This is a finding consistent with other studies (Lauer et al., 1982; Palmer

Table 1. Analysis by species.

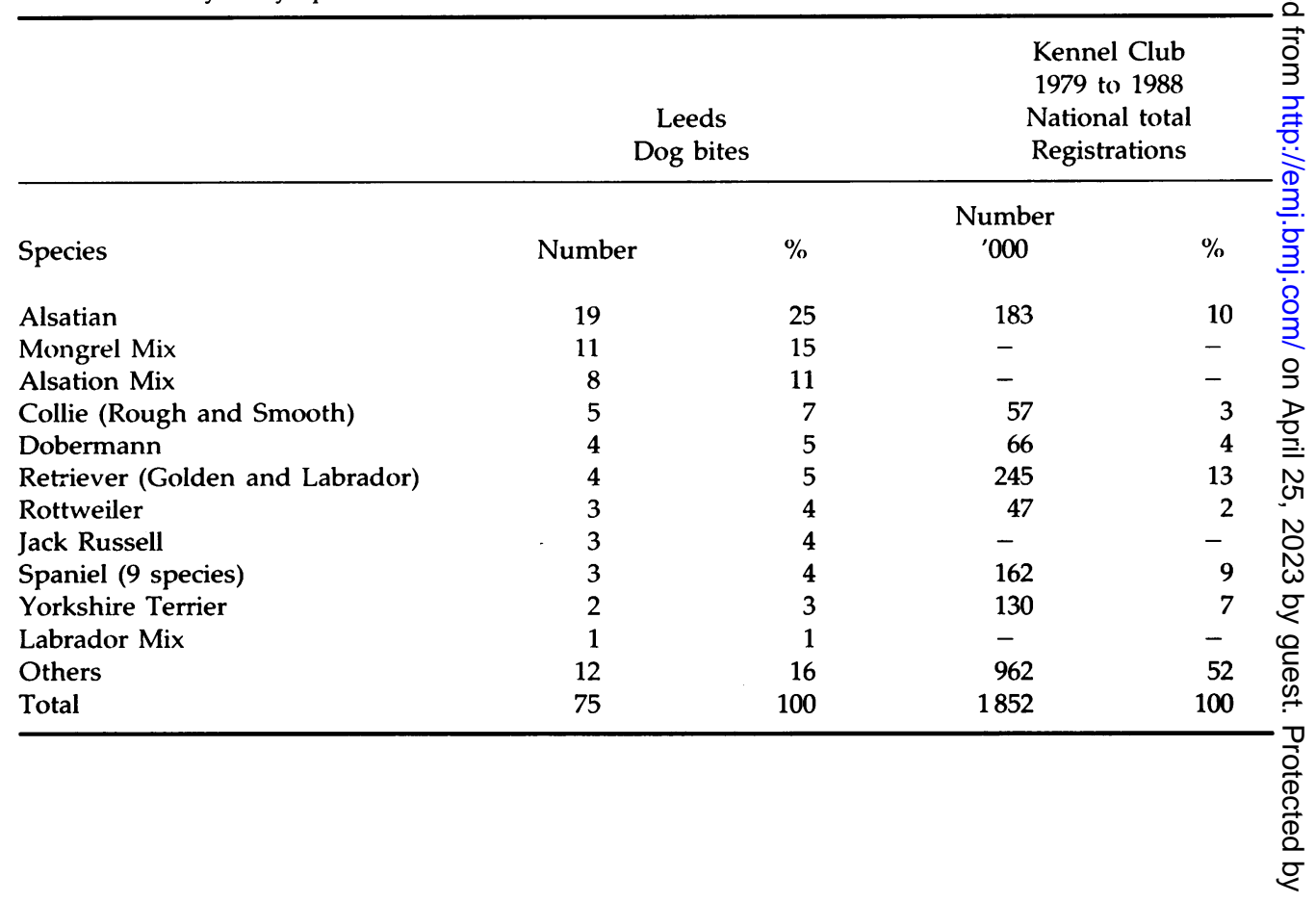


Rees 1983). Children under 11 years old often know or have seen the dog that bites them ( 20 out of 24 children in this study).

Alsatian or Alsatian mixes were the biggest group causing dog bites in this study (36\%). In 1988 Alsatians were the second most popular registered dog by the Kennel Club, and the second most popular species in the total registrations 1979 to 1988 (Kennel Guzette, 1989). Lauer et al. (1982) concluded that Alsatian dogs caused more bites than expected relative to their popularity as pets (Lauer et al., 1982).

There were 19 Alsatian bites of which $15(79 \%)$ were claimed to the unprovoked. This would seem to support the view that Alsatians are an unpredictable species, for unprovoked bites from other breeds combined were 21 of 56 bites (37\% of their total).

Rottweilers accounted for $3(4 \%)$ and Dobermanns for $4(4 \%)$ of all dog bites in this study. These species were ranked 6th and 11th respectively in the 1988 Kennel Gazette new registration listing, and accounted for $2 \%$ and $4 \%$ respectively of the total registrations for the 1979 to 1988 period (Table 1).

The most popular registered species in the 1988 Kennel Gazette listing and in the total registrations 1979 to 1988 were Retrievers (Labrador and Golden) (Table). Despite this Retrievers only caused $3(4 \%)$ of all bites, which would tend to support the view that Retrievers are of a placid nature.

It would seem that young children are a particular risk group for dog bites, a point worth considering before acquiring a family pet.

\section{REFERENCES}

Her Majesty's Stationery Office (1989) Monthly Digest of Statistics 19(Dec).

Lauer E. A., White W. C. \& Lauer B. A. (1982) Dog Bites. A Neglected Problem in Accident Prevention. American Journal of Diseases of Children 136, 202-4.

Palmer J., Rees M. (1983) Dog Bites of the Face; A 15 Year Review. British Journal of Plastic Surgery 36, 315-8.

Kennel Gazette (1989) April, 111 (1308) 75-7. 